\title{
L-Arginine polymers enhance coronary flow and reduce oxidative stress following cardiac transplantation in rats
}

\author{
Murray H. Kown, MDa \\ Maarten A. Lijkwan, BS ${ }^{a}$ \\ Christina L. Jahncke, BS ${ }^{\mathrm{a}}$ \\ Seiichiro Murata, MD, $\mathrm{PhD}^{\mathrm{a}}$ \\ Jonathan B. Rothbard, $\mathrm{PhD}^{\mathrm{b}}$ \\ Robert C. Robbins, MD
}

Background: Hearts treated with L-arginine polymers have demonstrated upregulated production of nitric oxide. The current study examined whether these polymers improved coronary flow and reduced myocardial oxidative stress after rat heart transplantation.

Methods: PVG donor hearts were incubated ex vivo with either $100 \mu \mathrm{mol} / \mathrm{L}$ L-arginine polymers 9 amino acids in length $(\mathrm{R} 9)(\mathrm{n}=7)$ or phosphate-buffered saline $(n=7)$ for 30 minutes after arrest and then transplanted heterotopically into the abdomen of ACI recipient rats. Coronary flows were assessed using fluorescent microspheres both at baseline (30 minutes after reperfusion) and at 6 hours and compared using the paired Student $t$ test. Evidence of oxidative stress was assessed in a separate cohort of similarly treated animals by enzyme-linked imunosorbent assay for rat tumor necrosis factor- $\alpha$ at 6 hours.

Results: Histochemistry with biotinylated L-arginine polymers demonstrated uptake of R9 into the vascular walls of treated allografts. Although all hearts experienced deterioration in coronary flow between baseline and 6 hours, the R9-treated group had a smaller reduction $(29.9 \%, P=.10)$ than the phosphate-buffered saline control group $(58.0 \%, P=.003)$. Tumor necrosis factor- $\alpha$ levels were also significantly reduced in the R9 treatment group compared with the phosphate-buffered saline category $(160 \pm 30$ versus $205 \pm 38, P=.007)$.

Conclusion: Rat cardiac allografts treated with R9 at the time of procurement exhibited less deterioration in coronary flow and a reduction in myocardial oxidative stress than the phosphate-buffered saline control group in the perioperative period. The use of arginine polymers may thus provide important myocardial protection against ischemia-reperfusion injury in both transplant and routine cardiac surgery cases.

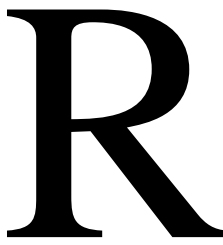

eperfusion of previously ischemic cardiac tissue leads to the generation of oxygen free radicals such as superoxide anions and hydroxyl groups, which in turn cause lipid peroxidation and cellular death. ${ }^{1}$ These cytotoxic reactive oxygen species initiate an inflammatory response that extends myocardial injury and impairs overall cardiac function beyond that caused by ischemia alone. ${ }^{2}$

Nitric oxide (NO) has long been recognized as a potential cardioprotective agent. In addition to its vasodilatory properties, it is a known free radical scavenger ${ }^{3}$ as well as inhibitor of neutrophilic adherence to the vascular endothelium. ${ }^{4,5}$ NO's ability to both attenuate neutrophil chemotaxis as well as scavenge the destructive by-products of its inflammatory mediators makes it an attractive therapeutic modality for cardiovascular procedures. 
NO is formed enzymatically by the action of NO synthase on its substrate, L-arginine. ${ }^{6}$ Various strategies have been utilized to administer this molecule in experimental models including providing specific $\mathrm{NO}$ donors, ${ }^{7}$ gene transfer of inducible NO synthase, ${ }^{8}$ and exogogenous Larginine. ${ }^{9}$ Recently, it was discovered that polymers of L-arginine of 6 amino acids (R6) in length or greater were highly effective in gaining intracellular access. These polymers thus represent a novel method of L-arginine delivery to biologic systems. Polymers of less than 6 amino acids were found to be ineffective in crossing cellular membranes. ${ }^{10}$ Previous experiments in this laboratory indeed found that treatment of cardiac allografts with L-arginine polymers of 9 amino acids in length (R9) resulted in cellular uptake as well as upregulation of NO production in these tissues. These findings were not found in those hearts treated with shorter L-arginine polymers of 5 amino acids in length. ${ }^{11}$

The objective of the current study was thus to determine whether similar treatment of donor hearts with R9 would increase coronary vasodilatation as assessed by regional blood flow measures as well as decrease parameters of reperfusion injury as measured by tumor necrosis factor (TNF)- $\alpha$ levels during the perioperative period. Myocardial cells have been shown to synthesize and release TNF- $\alpha$ during ischemia-reperfusion injury. ${ }^{12,13}$

\section{Methods}

\section{Peptide Synthesis}

L-Arginine polymers were synthesized on an Applied Biosystems 433 peptide synthesizer using previously described solid phase techniques and commercially available reagents (Applied Biosystems, Foster City, Calif) ${ }^{14}$ and provided as a gift by Cellgate Incorporated (Sunnyvale, Calif). The following sequences were used: R9: $\mathrm{NH}_{2}-\mathrm{RRRRRRRRR-CONH} \mathrm{C}_{2}(\mathrm{R}=\mathrm{L}$-arginine $)$ and phosphate-buffered saline (PBS). Biotinylated polymers were used in studies assessing translocation of the agent to the vascular walls of treated hearts.

\section{Animals}

Adult pathogen-free male ACI $\left(\mathrm{RT}^{\mathrm{a}}\right)$ and $\mathrm{PVG}\left(\mathrm{RT}^{\mathrm{c}}\right)$ rats weighing approximately $250 \mathrm{~g}$ were purchased from Harlan Sprague Dawley (Indianapolis, Ind) and housed at the animal care facilities at Stanford University Medical Center (Stanford, Calif). They were provided rat chow and water ad libitum and kept under standard temperature, humidity, and timed lighting conditions. Animals were treated in a humane manner in compliance with the "Guide for the Care and Use of Laboratory Animals" (National Institutes of Health publication no. 86-23, revised 1985).

\section{Heterotopic Heart Transplantation}

Intraabdominal heterotopic cardiac grafting was performed using a modification of the Ono and Lindsey technique. ${ }^{15}$ Animals were anesthetized by inhalation of $1 \%$ to $2 \%$ methoxyfluorane followed by intraperitoneal injection of pentobarbital $(40 \mathrm{mg} / \mathrm{kg})$. Heart and respiratory rates were closely monitored. After arrest of the PVG donor hearts with Stanford Cardioplegia (sodium bicarbonate, 25 $\mathrm{mEq} / \mathrm{L}$; potassium chloride, $30 \mathrm{mEq} / \mathrm{L} ; 5 \%$ dextrose; and $1.3 \%$ mannitol), $500 \mu \mathrm{L}$ of randomly assigned PBS $(\mathrm{n}=7)$ or 100 $\mu \mathrm{mol} / \mathrm{L}$ R9 $(\mathrm{n}=7)$ were administered proximal to an aortic crossclamp and then incubated within the hearts for 30 minutes in a $4^{\circ} \mathrm{C}$ PBS bath. A midline laparotomy was made in intubated recipient ACI rats, and the donor heart ascending aorta and pulmonary artery were anastamosed in an end-to-side fashion to the recipient's abdominal aorta and vena cava, respectively. The midline laparotomy incisions were then closed, and the animals underwent left lateral thoracotomy exposing the left atrium.

\section{Regional Myocardial Blood Flow Calculations}

Fifteen-micron fluorescent microspheres were used to calculate myocardial regional blood flow (RBF) as previously described. ${ }^{16}$ Approximately 500,000 spheres (NuFlow; IMT Technologies, Ltd, Irvine, Calif) were injected into the left atrium 30 minutes after unclamping and reperfusion of cardiac allografts while a reference blood sample was drawn for 60 seconds from the left femoral artery at a set rate.

The animals were then extubated and monitored closely for signs of distress for a 6-hour period. After this interval, they were reintubated and their thoracotomy incisions reopened. Fluorescent microspheres of a different color were then injected in a similar fashion as described above, after which the cardiac allografts were procured and the animals killed. Processing of tissues and counting of microspheres were performed in a blinded fashion by Interactive Medical Technologies, Ltd (Irvine, Calif). Briefly, tissues were digested in an alkaline reagent (catalog no. 501-105) overnight. The samples were then centrifuged at $1500 \mathrm{~g}$ after which the supernatant was collected, mixed with a microsphere counting reagent (catalog no. 501-107), and sonicated. The effluent was then passed through a $50-\mu \mathrm{m}$ filter and centrifuged, and the supernatant aspirated to leave a volume of $200 \mu \mathrm{L}$. The sample was then briefly sonicated and analyzed by flow cytometry. RBF was calculated according to the following equation: $Q i(\mathrm{~mL} / \mathrm{min})=$ $\left(f l_{i} / f l_{r e f}\right) * R(\mathrm{~mL} / \mathrm{min})$, where $Q i=$ flow to each segment of myocardium, $f l_{i}=$ fluorescence count of each piece, $f l_{\text {ref }}=$ fluorescence count of $\mathrm{RBF}$, and $R(\mathrm{~mL} / \mathrm{min})=$ reference blood withdrawal rate.

\section{Measurement of TNF- $\alpha$ Levels}

Additional allografts were treated with $100 \mu \mathrm{mol} / \mathrm{L} \mathrm{R} 9(\mathrm{n}=11)$ or PBS $(\mathrm{n}=10)$ and transplanted heterotopically as described above to provide tissues samples for TNF- $\alpha$ assays. As in the RBF studies, procurement was performed 6 hours after reperfusion of the allografts. Fresh tissue samples were homogenized in normal saline and centrifuged at $4^{\circ} \mathrm{C}$ for 20 minutes at $10,000 \mathrm{~g}$. The supernatant was then collected and stored at $-20^{\circ} \mathrm{C}$. TNF- $\alpha$ levels in these supernatant samples were measured by enzyme-linked immunoassay technique using a commercially available detection kit (Biosource International, Camarillo, Calif) and standardized to total protein concentrations determined utilizing a modification of the Lowry method (Sigma Diagnostics, St Louis, Mo).

\section{Confirmation of Translocation}

To demonstrate the ability of R9 penetration of vascular cells, PVG hearts were isolated as above and incubated for 30 minutes 

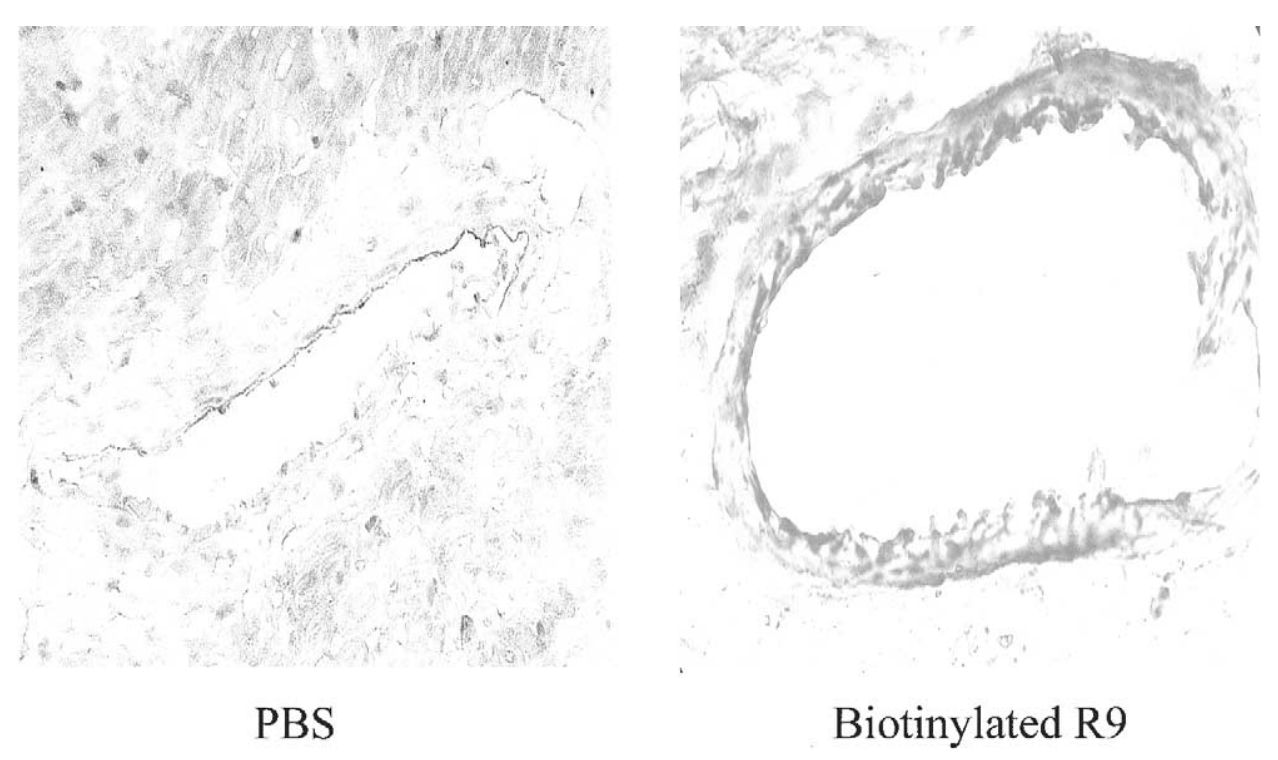

Figure 1. Representative cross sections at $400 \times$ of rat hearts treated ex vivo with either PBS or $100 \mu \mathrm{mol} / \mathrm{L}$ biotinylated R9 for $\mathbf{3 0}$ minutes. Areas of R9 uptake are indicated by nuclei darkened by 3,3'-diaminobenzidine staining of streptavidin-biotin complexes. Note in the PBS-treated group, only the methyl green counterstaining of nuclei are visible within the vascular wall.

with either PBS $(n=1)$ or $100 \mu \mathrm{mol} / \mathrm{L}(\mathrm{n}=1)$ biotinylated R9. The hearts were then flushed with PBS solution before harvesting, freezing in optimal cutting temperature (OCT) compound (TissueTek, Torrance, Calif) and cutting into $5-\mu \mathrm{m}$ sections. Slides were fixed in acetone for 10 minutes at $-20^{\circ} \mathrm{C}$, washed in PBS for 3 separate 5-minute intervals, and incubated for 30 minutes with peroxidase suppressor (Pierce, Rockford, Ill) to block endogenous activity. The slides were then washed in PBS as above and incubated with $5 \mu \mathrm{g} / \mathrm{mL}$ of horseradish peroxidase-conjugated streptavidin (Pierce) for 30 minutes. They were then washed and incubated for 60 seconds with the horseradish peroxidase substrate, 3,3'-diaminobenzidine (Sigma Diagnostics). Reactions were terminated by rinsing slides in distilled water before methyl green counterstaining and dehydration in ethanol. Slides were photographed under $400 \times$ magnification.

\section{Statistical Analysis}

All intra-animal and interanimal comparisons were made with the paired and unpaired Student $t$ test, respectively. $P$ values $<.05$ were considered significant.

\section{Results}

L-Arginine Polymer Uptake

Representative photomicrographs at $400 \times$ of hearts treated for 30 minutes with either PBS or biotinylated R9 demonstrated uptake of the latter within the coronary artery vasculature. The PBS-treated hearts showed the methyl green counterstaining of nuclei within the cells of the tunica intima and media layers, whereas the R9-treated hearts demonstrated brown staining indicating the presence of the biotinylated polymers (Figure 1).

\section{Regional Myocardial Blood Flow}

The cardiac allografts demonstrated deterioration in coronary flows as measured by fluorescent microspheres between the baseline and 6-hour time points. In those hearts pretreated with R9 polymers, however, the deterioration was only $29.9 \%$ and was not found to be statistically significant $(P=.10)$. PBS-treated hearts, on the other hand, showed a statistically significant $58.0 \%$ decline in RBF between the baseline and 6-hour time points $(P=.003)$. Mean RBFs for both PBS and R9 groups are shown in Figure 2.

\section{Measurement of TNF- $\alpha$ Levels}

TNF- $\alpha$ levels as measured by enzyme-linked immunosorbent assay were significantly lower in the R9-pretreated cardiac allografts at the 6-hour time point (160 \pm 30 versus $205 \pm 38 \mathrm{pg} / \mathrm{mL}$ for the R9 and PBS groups, respectively; $P=.007)$ (Figure 3).

\section{Discussion}

Our initial studies utilizing polyarginine therapy in a cardiac transplant model found an $89 \%$ transfection efficiency of vascular endothelial cells associated with a $30 \%$ upregulation in the production of NO. ${ }^{11}$ The observation that polymers of L-arginine gain intracellular access was first theorized studying the transactivator protein of the human immunodeficiency virus (tat), which is rich in its concentration of this amino acid, ${ }^{17,18}$ and confirmed with the work 


\section{Regional Blood Flow}

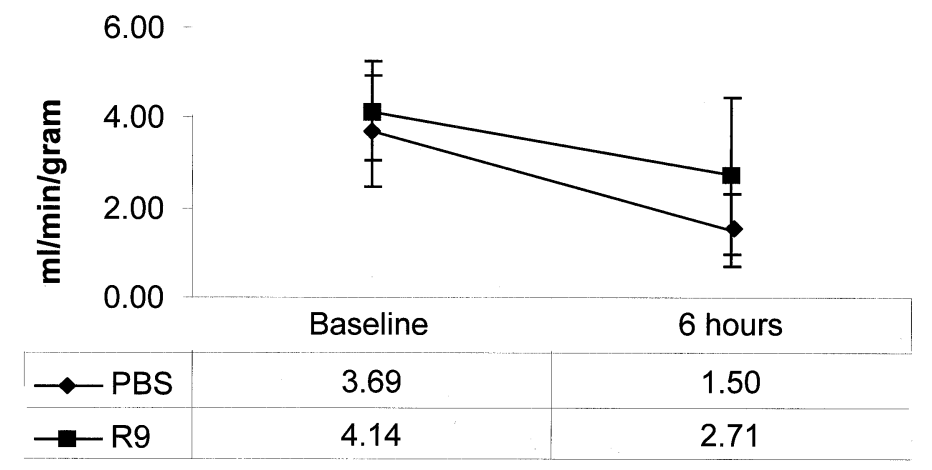

Figure 2. Mean RBFs at baseline and 6 hours for both PBS and R9 treatment groups.

\section{TNF- $\alpha$ Levels}

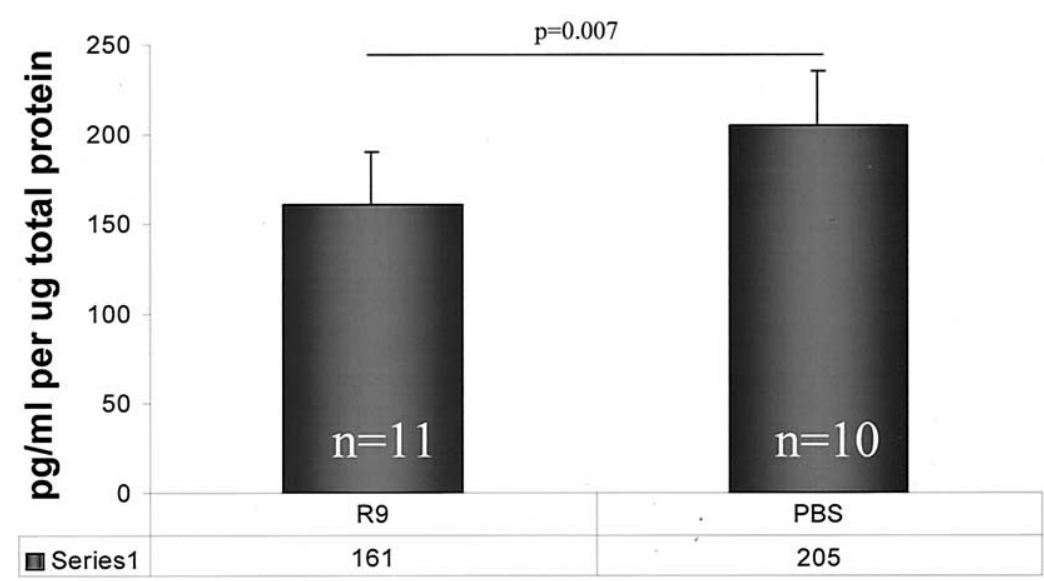

Figure 3. TNF- $\alpha$ expression is significantly less at 6 hours postreperfusion in the R9 group compared with the PBS-treated animals.

of Uemura and colleagues, ${ }^{19}$ who described the uptake of these polymers in vascular ring segments.

In our original study, chronic rejection as manifested by graft coronary artery disease (GCAD) was found to be significantly reduced in L-arginine polymer-treated allografts at both 60 and 90 days posttransplantation. Although NO is thought to limit the development of GCAD by inhibiting vascular smooth muscle cell proliferation within the neointimal layer, ${ }^{20}$ it was conjectured that there may have also been a contribution from its protective effects on graft reperfusion injury. GCAD, while primarily immunologically driven, is known to be exacerbated by early ischemic injury. ${ }^{21}$ The current study was thus performed to examine the ability of L-arginine polymer therapy to inhibit cardiac injury associated with graft reperfusion as well as document any increase in regional blood flow due to NO's vasodilatory properties.

Coronary vasospasm after cardiopulmonary bypass may be a significant cause of postoperative cardiac dysfunction and even death. ${ }^{22}$ In many situations, the resulting systemic hypotension renders the use of traditional vasodilating agents infeasible. Although direct intracardiac or intracoronary injections of nitroglycerin or calcium channel-blocking agents have been effective in reversing coronary artery spasm, ${ }^{23,24}$ the difficulty of these routes of delivery makes these methods suboptimal as well.

L-Arginine polymer treatment of hearts undergoing cardiac arrest potentially circumvents this problem by provid- 
ing a means of continuous delivery of NO to the coronary bed during the perioperative period. The previous study's measurement of 30\% elevation in NO levels was performed at 24 hours of postreperfusion. Although the current study looked at only the 6-hour time point in terms of RBFs, it is thus feasible that the beneficial effects of NO on coronary vasomotor tone would have been present for a longer duration during the perioperative period.

Many investigators have utilized L-arginine monomers to upregulate NO in their biologic models. ${ }^{25-27}$ Polymers confer the specific advantage over single amino acids in that there is delivery of multiple units of L-arginine to act as substrates for NO synthase. In addition, polyarginine utilizes an independent active transport mechanism distinct from that used by the single amino acids that leads to an approximate 100 -fold increase in its rate of uptake. ${ }^{28}$

The beneficial effects of $\mathrm{L}$-arginine have been related to the upregulation of NO with subsequent inhibition of neutrophil adherence ${ }^{29,30}$ and clearance of its free radical byproducts. ${ }^{31}$ The data in this current study showed a definite reduction in myocardial injury as manifested by TNF- $\alpha$ production. Although TNF- $\alpha$ is released by injured myocardium, it is not a mere marker for reperfusion injury. It is a cytokine that further contributes to the process of injury by autoregulating its own production as well as recruiting other inflammatory mediators such as interleukin- $1 .{ }^{32}$ Its effects thus include decreased myocardial contractility, decreased systemic vascular resistance, and resulting hypotension. ${ }^{13}$ Thus, although the reduced TNF- $\alpha$ levels seen with R9 treatment in this study may be indicative of decreased reperfusion injury, there may have also been a potential additional therapeutic benefit associated with the reduction of this rather deleterious inflammatory mediator.

This study did not look specifically at cardiac function, although it is logical to consider whether the reduction of vasospasm coupled with the protective effects against reperfusion injury would lead to improved perioperative graft function. Future studies will thus consider functional parameters such as $d p / d t$ or the load independent measure of contractility, the preload recruitable stroke work index, to evaluate whether R9 therapy elicits any improvements in these endpoints as well. There were no graft failures during the 6-hour study period in either the PBS or R9 groups. Future studies may look at increasing the ischemic times before reperfusion until such a period when graft function is compromised. R9 therapy can then be compared against these controls to access whether there are any improvements in function or survival. Another possibility is to access RBF, TNF- $\alpha$ production, and overall function at increasing time intervals from surgery. In this way, benefit can be clearly documented throughout the immediate postoperative period.
In conclusion, treatment of cardiac allografts with Larginine polymers before transplantation resulted in a reduction of deterioration in coronary perfusion as measured by RBFs as well as decreased evidence of reperfusion injury as accessed by TNF- $\alpha$ levels. Although the model is one of transplantation, the findings may be relevant to all cardiovascular surgeries where cardiopulmonary bypass implies an inherent ischemic time followed by reperfusion. Future studies will assess whether the mitigation of ischemiareperfusion injury and the improvement in coronary blood flows with L-arginine polymer therapy will result in functional improvements during the perioperative period.

\section{References}

1. Masini E, Salvemini D, Ndisang JF, et al. Cardioprotective activity of endogenous and exogenous nitric oxide on ischaemia reperfusion injury in isolated guinea pig hearts. Inflamm Res. 1999;48:561-8.

2. Park JL, Lucchesi BR. Mechanisms of myocardial reperfusion injury. Ann Thorac Surg. 1999;68:1905-12.

3. Rubanyi GM, Ho EH, Cantor EH, Lumma WC, Botelho LH. Cytoprotective function of nitric oxide: inactivation of superoxide radicals produced by human leukocytes. Biochem Biophys Res Commun. 1991; 181:1392-7.

4. Vinten-Johansen J, Sato H, Zhao ZQ. The role of nitric oxide and NO-donor agents in myocardial protection from surgical ischemicreperfusion injury. Int J Cardiol. 1995;50:273-81.

5. Pabla R, Buda AJ, Flynn DM, et al. Nitric oxide attenuates neutrophilmediated myocardial contractile dysfunction after ischemia and reperfusion. Circ Res. 1996;78:65-72.

6. Goumas G, Tentolouris C, Tousoulis D, Stefanadis C, Toutouzas P. Therapeutic modification of the L-arginine-eNOS pathway in cardiovascular diseases. Atherosclerosis. 2001;154:255-67.

7. Salas A, Gironella M, Soriano A, et al. Nitric oxide supplementation ameliorates dextran sulfate sodium-induced colitis in mice. Lab Invest. 2002;82:597-607.

8. von der Leyen HE, Gibbons GH, Morishita R, et al. Gene therapy inhibiting neointimal vascular lesion: in vivo transfer of endothelial cell nitric oxide synthase gene. Proc Natl Acad Sci U S A. 1995;92: $1137-41$

9. Okazaki J, Komori K, Kawasaki K, Eguchi D, Ishida M, Sugimachi K. L-Arginine inhibits smooth muscle cell proliferation of vein graft intimal thickness in hypercholesterolemic rabbits. Cardiovasc Res. 1997;36:429-36.

10. Mitchell DJ, Kim DT, Steinman L, Fathman CG, Rothbard JB. Polyarginine enters cells more efficiently than other polycationic homopolymers. J Pept Res. 2000;56:318-25.

11. Kown $\mathrm{MH}$, van der Steenhoven $\mathrm{T}$, Uemura $\mathrm{S}$, et al. L-Arginine polymer mediated inhibition of graft coronary artery disease after cardiac transplantation. Transplantation. 2001;71:1542-8.

12. Gurevitch J, Frolkis I, Yuhas Y, et al. Tumor necrosis factor-alpha is released from the isolated heart undergoing ischemia and reperfusion. J Am Coll Cardiol. 1996;28:247-52.

13. Meldrum DR. Tumor necrosis factor in the heart. Am J Physiol. 1998;274:R577-95.

14. Hill CM, Liu A, Marshall KW, et al. Exploration of requirements for peptide binding to HLA DRB1*0101 and DRB1*0401. J. Immunol. 1994;152:2890-8.

15. Ono K, Lindsey ES. Improved technique of heart transplantation in rats. J Thorac Cardiovasc Surg. 1969;57:225-9.

16. Kobayashi N, Kobayashi K, Kouno K, Horinaka S, Yagi S. Effects of intra-atrial injection of colored microspheres on systemic hemodynamics and regional blood flow in rats. Am J Physiol. 1994;266:H1910-7.

17. Efthymiadis A, Briggs LJ, Jans DA. The HIV-1 Tat nuclear localization sequence confers novel nuclear import properties. J Biol Chem. 1998;273:1623-8.

18. Vives E, Brodin P, Lebleu B. A truncated HIV-1 Tat protein basic 
domain rapidly translocates through the plasma membrane and accumulates in the cell nucleus. J Biol Chem. 1997;272:16010-7.

19. Uemura S, Fathman CG, Rothbard JB, Cooke JP. Rapid and efficient vascular transport of arginine polymers inhibits myointimal hyperplasia. Circulation. 2000;102:2629-35.

20. Lloyd-Jones DM, Bloch KD. The vascular biology of nitric oxide and its role in atherogenesis. Аnпи Rev Med. 1996;47:365-75.

21. Knight RJ, Dikman S, Liu H, Martinelli GP. Cold ischemic injury accelerates the progression to chronic rejection in a rat cardiac allograft model. Transplantation. 1997;64:1102-7.

22. Lemmer JHJ, Kirsh MM. Coronary artery spasm following coronary artery surgery. Ann Thorac Surg. 1988;46:108-15.

23. Jost S, Sturm M, Hausmann D, Lippolt P, Lichtlen PR. Standardization of coronary vasomotor tone with intracoronary nitroglycerin. Am J Cardiol. 1996;78:120-3.

24. Ishihara M, Sato H, Tateishi H, et al. Effects of various doses of intracoronary diltiazem on coronary resistance vessels in humans. Jpn Circ J. 1995;59:790-8.

25. Agullo L, Garcia-Dorado D, Inserte J, et al. L-Arginine limits myocardial cell death secondary to hypoxia-reoxygenation by a cGMPdependent mechanism. Am J Physiol. 1999;276:H1574-80.
26. Mizuno T, Watanabe M, Sakamoto T, Sunamori M. L-Arginine, a nitric oxide precursor, attenuates ischemia-reperfusion injury by inhibiting inositol-1,4,5-triphosphate. J Thorac Cardiovasc Surg. 1998; 115:931-6.

27. Yan Y, Davani S, Chocron S, Kantelip B, Muret P, Kantelip JP. Effects of L-arginine administration before cardioplegic arrest on ischemia-reperfusion injury. Ann Thorac Surg. 2001;72:1985-90.

28. Mitchell DJ, Kim DT, Steinman L, Fathman CG, Rothbard JB. Polyarginine enters cells more efficiently than other polycationic homopolymers. J Pept Res. 2000;56:318-25.

29. Kibbe M, Billiar T, Tzeng E. Inducible nitric oxide synthase and vascular injury. Cardiovasc Res. 1999;43:650-7.

30. Weyrich AS, Ma XL, Lefer AM. The role of L-arginine in ameliorating reperfusion injury after myocardial ischemia in the cat. Circulation. 1992;86:279-88.

31. Lass A, Suessenbacher A, Wolkart G, Mayer B, Brunner F. Functional and analytical evidence for scavenging of oxygen radicals by Larginine. Mol Pharmacol. 2002;61:1081-8.

32. Dinarello CA, Cannon JG, Wolff SM, et al. Tumor necrosis factor (cachectin) is an endogenous pyrogen and induces production of interleukin 1. J Exp Med. 1986;163:1433-50.

\section{Authoritative}

The Journal of Thoracic and Cardiovascular Surgery is the most frequently cited thoracic/cardiovascular surgery journal in the Science Citation Index. An article in JTCVS is cited on average almost twice as often as those in the closest cardiothoracic journal. 